\title{
New code upper bounds from the Terwilliger algebra and semidefinite programming
}

\author{
Alexander Schrijver
}

\begin{abstract}
We give a new upper bound on the maximum size $A(n, d)$ of a binary code of word length $n$ and minimum distance at least $d$. It is based on block-diagonalising the Terwilliger algebra of the Hamming cube. The bound strengthens the Delsarte bound, and can be calculated with semidefinite programming in time bounded by a polynomial in $n$. We show that it improves a number of known upper bounds for concrete values of $n$ and $d$.

From this we also derive a new upper bound on the maximum size $A(n, d, w)$ of a binary code of word length $n$, minimum distance at least $d$, and constant weight $w$, again strengthening the Delsarte bound and yielding several improved upper bounds for concrete values of $n, d$, and $w$.
\end{abstract}

Index Terms-block-diagonalisation, codes, constant-weight codes, Delsarte bound, semidefinite programming, Terwilliger algebra, upper bounds.

\section{DESCRIPTION OF THE METHOD}

We present a new upper bound on $A(n, d)$, the maximum size of a binary code of word length $n$ and minimum distance at least $d$. The bound is based on block-diagonalising the (noncommutative) Terwilliger algebra of the Hamming cube and on semidefinite programming. The bound refines the Delsarte bound [4], which is based on diagonalising the (commutative) Bose-Mesner algebra of the Hamming cube and on linear programming. We describe the approach in this section, and go over to the details in Section II.

Taking a tensor product of the algebra, this approach also yields a new upper bound on $A(n, d, w)$, the maximum size of a binary code of word length $n$, minimum distance at least $d$, and constant weight $w$. This bound strengthens the Delsarte bound for constant-weight codes. We describe this method in Section III.

Fix a nonnegative integer $n$, and let $\mathcal{P}$ be the collection of all subsets of $\{1, \ldots, n\}$. We identify code words in $\{0,1\}^{n}$ with their support. So a code $C$ is a subset of $\mathcal{P}$. The Hamming distance of $X, Y \in \mathcal{P}$ is equal to $|X \triangle Y|$. The minimum distance of a code $C$ is the minimum Hamming distance of distinct elements of $C$. For finite sets $U$ and $V$, a $U \times V$ matrix is a matrix whose rows and columns are indexed by $U$ and $V$, respectively.

For background on coding theory and association schemes we refer to MacWilliams and Sloane [9]. However, most of this paper is self-contained. While we will mention below a theorem on the existence of a block-diagonalisation of a $\mathrm{C} *-$ algebra, we prove this theorem for the algebras concerned by displaying an explicit block-diagonalisation.

CWI and University of Amsterdam. Mailing address: CWI, Kruislaan 413, 1098 SJ Amsterdam, The Netherlands. Email: lex@cwi.nl

\section{A. The Terwilliger algebra}

We first describe the Terwilliger algebra of the Hamming cube, in a form convenient for our purposes. For background we refer to our notes in Subsection I-C.

For nonnegative integers $i, j, t$, let $M_{i, j}^{t}$ be the $\mathcal{P} \times \mathcal{P}$ matrix with

$$
\left(M_{i, j}^{t}\right)_{X, Y}:= \begin{cases}1 & \text { if }|X|=i,|Y|=j,|X \cap Y|=t \\ 0 & \text { otherwise }\end{cases}
$$

for $X, Y \in \mathcal{P}$. So $\left(M_{i, j}^{t}\right)^{\top}=M_{j, i}^{t}$. It is trivial but useful to note that if $|X|=i$ and $|Y|=j$, then $|X \cap Y|=t$ is equivalent to $|X \triangle Y|=i+j-2 t$.

Let $\mathcal{A}_{n}$ be the set of matrices

$$
\sum_{i, j, t=0}^{n} x_{i, j}^{t} M_{i, j}^{t}
$$

with $x_{i, j}^{t} \in \mathbb{C}$. It is easy to check that $\mathcal{A}_{n}$ is a $C *$-algebra: it is closed under addition, scalar and matrix multiplication, and taking the adjoint. (Matrix multiplication follows from the fact that $M_{i, j}^{t} M_{j^{\prime}, k}^{s}=\mathbf{0}$ if $j \neq j^{\prime}$, and that for $X, Z \in \mathcal{P}$ then the number of $Y \in \mathcal{P}$ with $|Y|=j,|X \cap Y|=t,|Y \cap Z|=s$ only depends on $|X|,|Z|$, and $|X \cap Z|$. So $M_{i, j}^{t} M_{j, k}^{s}$ is a linear combination of $M_{i, k}^{u}$ for $u=0, \ldots, n$.)

This algebra is called the Terwilliger algebra [14] of the Hamming cube $H(n, 2)$. It has dimension

$$
\operatorname{dim} \mathcal{A}_{n}=\left(\begin{array}{c}
n+3 \\
3
\end{array}\right),
$$

since it is the number of triples $(i, j, t)$ with $M_{i, j}^{t} \neq \mathbf{0}$, which is equal to the number of triples $(a, b, t)$ with $a+b+t \leq n$.

Since $\mathcal{A}_{n}$ is a $\mathrm{C} *$-algebra and since $\mathcal{A}_{n}$ contains the identity matrix, there exists a unitary $\mathcal{P} \times \mathcal{P}$ matrix $U$ (that is, $U^{*} U=$ $I$ ) and positive integers $p_{0}, q_{0}, \ldots, p_{m}, q_{m}$ (for some $m$ ) such that $U^{*} \mathcal{A}_{n} U$ is equal to the collection of all block-diagonal matrices

$$
\left(\begin{array}{cccc}
C_{0} & 0 & \cdots & 0 \\
0 & C_{1} & \cdots & 0 \\
\vdots & \vdots & \ddots & 0 \\
0 & 0 & \cdots & C_{m}
\end{array}\right)
$$

(for later purposes, we start the numbering at 0 ), where each $C_{k}$ is a block-diagonal matrix with $q_{k}$ repeated, identical blocks of order $p_{k}$ : 
(5) $\quad C_{k}=\left(\begin{array}{cccc}B_{k} & 0 & \cdots & 0 \\ 0 & B_{k} & \cdots & 0 \\ \vdots & \vdots & \ddots & 0 \\ 0 & 0 & \cdots & B_{k}\end{array}\right)$.

So $p_{0}^{2}+\cdots+p_{m}^{2}=\operatorname{dim}\left(\mathcal{A}_{n}\right)=\left(\begin{array}{c}n+3 \\ 3\end{array}\right)$ and $p_{0} q_{0}+\cdots+p_{m} q_{m}=$ $2^{n}$.

By deleting copies of blocks, we see that $\mathcal{A}_{n}$ is (as a C*algebra) isomorphic to the direct sum

$$
\begin{aligned}
& \bigoplus_{k=0}^{m} \mathbb{C}^{p_{k} \times p_{k}}=\left\{\left(\begin{array}{cccc}
B_{0} & 0 & \cdots & 0 \\
0 & B_{1} & \cdots & 0 \\
\vdots & \vdots & \ddots & 0 \\
0 & 0 & \cdots & B_{m}
\end{array}\right) \mid B_{k} \in\right. \\
& \left.\mathbb{C}^{p_{k} \times p_{k}} \text { for } k=0, \ldots, m\right\} .
\end{aligned}
$$

The isomorphism maintains positive semidefiniteness of matrices. The number $m$ and the block sizes $p_{k}$ and block multiplicities $q_{k}$ are (up to permutation of the indices $k$ ) uniquely determined by the $\mathrm{C} *$-algebra.

So far, this is all standard $\mathrm{C} *$-algebra theory, but we will need this block-diagonalisation of the Terwilliger algebra $\mathcal{A}_{n}$ more explicitly. In Section II we will specify a matrix $U$ with the required properties. It will turn out that $U$ can be taken real, that $m=\left\lfloor\frac{n}{2}\right\rfloor$, and that, for $k=0, \ldots,\left\lfloor\frac{n}{2}\right\rfloor$, there is a block $B_{k}$ of order $p_{k}=n-2 k+1$ and multiplicity $q_{k}=\left(\begin{array}{l}n \\ k\end{array}\right)-\left(\begin{array}{c}n \\ k-1\end{array}\right)$. (One may check that indeed $\sum_{k=0}^{\left\lfloor\frac{n}{2}\right\rfloor}(n-2 k+1)^{2}=\left(\begin{array}{c}n+3 \\ 3\end{array}\right)$ (cf. (48) below) and $\sum_{k=0}^{\left\lfloor\frac{n}{2}\right\rfloor}\left(\left(\begin{array}{c}n \\ k\end{array}\right)-\left(\begin{array}{c}n \\ k-1\end{array}\right)\right)(n-2 k+1)=2^{n}$ (cf. (41) below).)

To describe the image of (2) in (6), define, for $i, j, k, t \in$ $\{0, \ldots, n\}$ :

$$
\beta_{i, j, k}^{t}:=\sum_{u=0}^{n}(-1)^{u-t}\left(\begin{array}{c}
u \\
t
\end{array}\right)\left(\begin{array}{c}
n-2 k \\
u-k
\end{array}\right)\left(\begin{array}{c}
n-k-u \\
i-u
\end{array}\right)\left(\begin{array}{c}
n-k-u \\
j-u
\end{array}\right) .
$$

In Theorem 1 (concluding Section II) we will see that, for $k=0, \ldots,\left\lfloor\frac{n}{2}\right\rfloor$, the $k$ th block $B_{k}$ of the image (6) of (2) is the following $(n-2 k+1) \times(n-2 k+1)$ matrix:

$$
\left(\sum_{t}\left(\begin{array}{c}
n-2 k \\
i-k
\end{array}\right)^{-\frac{1}{2}}\left(\begin{array}{c}
n-2 k \\
j-k
\end{array}\right)^{-\frac{1}{2}} \beta_{i, j, k}^{t} x_{i, j}^{t}\right)_{i, j=k}^{n-k} .
$$

\section{B. Application to coding}

Let $C \subseteq \mathcal{P}$ be any code. It will be convenient to assume $\emptyset \neq C \neq \mathcal{P}$.

Let $\Pi$ be the set of (distance-preserving) automorphisms $\pi$ of $\mathcal{P}$ with $\emptyset \in \pi(C)$, and let $\Pi^{\prime}$ be the set of automorphisms $\pi$ of $\mathcal{P}$ with $\emptyset \notin \pi(C)$. Let $\chi^{\pi(C)}$ denote the incidence vector of $\pi(C)$ in $\{0,1\}^{\mathcal{P}}$ (taken as column vector). Define the $\mathcal{P} \times \mathcal{P}$ matrices $R$ and $R^{\prime}$ by:

$$
\begin{aligned}
& R:=\sum_{\pi \in \Pi}|\Pi|^{-1} \chi^{\pi(C)}\left(\chi^{\pi(C)}\right)^{\top} \text { and } \\
& R^{\prime}:=\sum_{\pi \in \Pi^{\prime}}\left|\Pi^{\prime}\right|^{-1} \chi^{\pi(C)}\left(\chi^{\pi(C)}\right)^{\top} .
\end{aligned}
$$

As $R$ and $R^{\prime}$ are sums of positive semidefinite matrices, they are positive semidefinite. Moreover, $R$ and $R^{\prime}$ belong to $\mathcal{A}_{n}$. To see this, define

$$
x_{i, j}^{t}:=\frac{1}{|C|\left(\begin{array}{c}
n \\
i-t, j-t, t
\end{array}\right)} \lambda_{i, j}^{t}
$$

where $\left(\begin{array}{c}a \\ b_{1}, \ldots, b_{m}\end{array}\right)$ denotes the number of pairwise disjoint subsets of a set of size $a$, of sizes $b_{1}, \ldots, b_{m}$ respectively, and where

$$
\begin{aligned}
& \lambda_{i, j}^{t}:=\text { the number of triples }(X, Y, Z) \in C^{3} \text { with } \\
& |X \triangle Y|=i,|X \triangle Z|=j \text {, and }|(X \triangle Y) \cap(X \triangle Z)|=t .
\end{aligned}
$$

We set $x_{i, j}^{t}=0$ if $\left(\begin{array}{c}n \\ i-t, j-t, t\end{array}\right)=0$.

Then

\section{Proposition 1:}

$$
\begin{aligned}
& R=\sum_{i, j, t} x_{i, j}^{t} M_{i, j}^{t} \text { and } \\
& R^{\prime}=\frac{|C|}{2^{n}-|C|} \sum_{i, j, t}\left(x_{i+j-2 t, 0}^{0}-x_{i, j}^{t}\right) M_{i, j}^{t} .
\end{aligned}
$$

Proof: Consider any $X \in \mathcal{P}$, and let $\Pi_{X}$ be the set of automorphisms $\pi$ of $\mathcal{P}$ with $\pi(X)=\emptyset$. So $\left|\Pi_{X}\right|=n$ !. Let

$$
R_{X}:=\sum_{\pi \in \Pi_{X}}\left|\Pi_{X}\right|^{-1} \chi^{\pi(C)}\left(\chi^{\pi(C)}\right)^{\top} .
$$

As the value of $\left(R_{X}\right)_{Y, Z}$ only depends on $|X \triangle Y|,|X \triangle Z|$, and $|(X \triangle Y) \cap(X \triangle Z)|$, we know that $R_{X}$ belongs to $\mathcal{A}_{n}$. In fact,

$$
R_{X}=\sum_{i, j, t}\left(\begin{array}{c}
n \\
i-t, j-t, t
\end{array}\right)^{-1} \lambda_{i, j}^{t, X} M_{i, j}^{t}
$$

where $\lambda_{i, j}^{t, X}$ is the number of pairs $(Y, Z) \in C^{2}$ with $|X \triangle Y|=$ $i,|X \triangle Z|=j$, and $|(X \triangle Y) \cap(X \triangle Z)|=t$.

Equation (14) follows from the fact that for any $\pi \in \Pi_{X}$ and for all $i, j, t$, the number of 1's of $\chi^{\pi(C)}\left(\chi^{\pi(C)}\right)^{\top}$ in positions where $M_{i, j}^{t}$ is 1 , is equal to $\lambda_{i, j}^{t, X}$. As there are $\left(\begin{array}{c}n \\ i-t, j-t, t\end{array}\right)$ such positions, we obtain (14).

Now $R=\sum_{X \in C}|C|^{-1} R_{X}$ and $R^{\prime}=\sum_{X \in \mathcal{P} \backslash C}\left(2^{n}-\right.$ $|C|)^{-1} R_{X}$. Moreover,

$$
\sum_{X \in C} \lambda_{i, j}^{t, X}=\lambda_{i, j}^{t}
$$

and

$$
\sum_{X \in \mathcal{P} \backslash C} \lambda_{i, j}^{t, X}=\left(\begin{array}{c}
i+j-2 t \\
i-t
\end{array}\right)\left(\begin{array}{c}
n-i-j+2 t \\
t
\end{array}\right) \lambda_{i+j-2 t, 0}^{0}-\lambda_{i, j}^{t} .
$$

The latter expression follows from 


$$
\sum_{X \in \mathcal{P}} \lambda_{i, j}^{t, X}=\left(\begin{array}{c}
i+j-2 t \\
i-t
\end{array}\right)\left(\begin{array}{c}
n-i-j+2 t \\
t
\end{array}\right) \lambda_{i+j-2 t, 0}^{0},
$$

which holds since for any pair $(Y, Z) \in C^{2}$ with $|Y \triangle Z|=i+$ $j-2 t$, the number of sets $X \in \mathcal{P}$ with $|X \triangle Y|=i,|X \triangle Z|=$ $j$, and $|(X \triangle Y) \cap(X \triangle Z)|=t$ is equal to $\left(\begin{array}{c}i+j-2 t \\ i-t\end{array}\right)\left(\begin{array}{c}n-i-j+2 t \\ t\end{array}\right)$.

Since $|\Pi|=|C| n$ ! and $\left|\Pi^{\prime}\right|=\left(2^{n}-|C|\right) n$ !, and since

$$
\left.\left(\begin{array}{c}
n \\
i-t, j-t, t
\end{array}\right)\right)^{-1}\left(\begin{array}{c}
i+j-2 t \\
i-t
\end{array}\right)\left(\begin{array}{c}
n-i-j+2 t \\
t
\end{array}\right)=\left(\begin{array}{c}
n \\
i+j-2 t
\end{array}\right)^{-1}
$$

(14) gives (12).

The positive semidefiniteness of $R$ and $R^{\prime}$ is by (8) equivalent to:

for each $k=0, \ldots,\left\lfloor\frac{n}{2}\right\rfloor$, the matrices

$$
\left(\sum_{t=0}^{n} \beta_{i, j, k}^{t} x_{i, j}^{t}\right)_{i, j=k}^{n-k}
$$

and

$$
\left(\sum_{t=0}^{n} \beta_{i, j, k}^{t}\left(x_{i+j-2 t, 0}^{0}-x_{i, j}^{t}\right)\right)_{i, j=k}^{n-k}
$$

are positive semidefinite.

(We have deleted the factor $\left(\begin{array}{c}n-2 k \\ i-k\end{array}\right)^{-\frac{1}{2}}\left(\begin{array}{c}n-2 k \\ j-k\end{array}\right)^{-\frac{1}{2}}$ as it makes the coefficients integer, while positive semidefiniteness is maintained.)

The $x_{i, j}^{t}$ 's moreover satisfy the following constraints, where (iv) holds if $C$ has minimum distance at least $d$ :

$$
\begin{aligned}
& \text { (i) } x_{0,0}^{0}=1, \\
& \text { (ii) } 0 \leq x_{i, j}^{t} \leq x_{i, 0}^{0} \text { and } x_{i, 0}^{0}+x_{j, 0}^{0} \leq 1+x_{i, j}^{t} \text { for all } \\
& \\
& i, j, t \in\{0, \ldots, n\}, \\
& \text { (iii) } x_{i, j}^{t}=x_{i^{\prime}, j^{\prime}} \text { if }\left(i^{\prime}, j^{\prime}, i^{\prime}+j^{\prime}-2 t^{\prime}\right) \text { is a permutation } \\
& \\
& \text { of }(i, j, i+j-2 t), \\
& \text { (iv) } x_{i, j}^{t}=0 \text { if }\{i, j, i+j-2 t\} \cap\{1, \ldots, d-1\} \neq \emptyset .
\end{aligned}
$$

(Condition (ii) follows from the fact that each row of $R$ and $R^{\prime}$ is nonnegative and is dominated by its diagonal entry (by (9)). Conditions (iii) and (iv) follow from the fact that $\lambda_{i, j}^{t}$ is equal to the number of triples $(X, Y, Z)$ in $C^{3}$ with $|X \triangle Y|=i$, $|X \triangle Z|=j$, and $|Y \triangle Z|=i+j-2 t$, as follows directly from (11).)

Moreover,

$$
|C|=\sum_{i=0}^{n}\left(\begin{array}{l}
n \\
i
\end{array}\right) x_{i, 0}^{0},
$$

since $|C|^{2}=\sum_{i=0}^{n} \lambda_{i, 0}^{0}$. Hence we obtain an upper bound on $A(n, d)$ by considering the $x_{i, j}^{t}$ as variables, and by

(22) maximizing $\sum_{i=0}^{n}\left(\begin{array}{c}n \\ i\end{array}\right) x_{i, 0}^{0}$ subject to conditions (19) and (20).
This is a semidefinite programming problem with $O\left(n^{3}\right)$ variables, and it can be solved in time polynomial in $n$. (A generic form of a semidefinite programming problem is: given $c_{1}, \ldots, c_{t} \in \mathbb{R}$ and real symmetric matrices $A_{0}, \ldots, A_{t}, B$ (of equal dimensions), find $x_{1}, \ldots, x_{t} \in \mathbb{R}$ that maximize $\sum_{i} c_{i} x_{i}$ subject to the condition that $\left(\sum_{i} x_{i} A_{i}\right)-B$ is positive semidefinite. If all $A_{i}$ and $B$ are diagonal matrices, we have a linear programming problem. Under certain conditions (which are satisfied in the present case), semidefinite programming problems can be solved in polynomial time. For background on semidefinite programming we refer to Todd [15] and Wright [17].)

One may reduce the number of variables by using the wellknown facts that if $d$ is odd then $A(n, d)=A(n+1, d+1)$ and that if $d$ is even then $A(n, d)$ is attained by a code with all code words having even Hamming weights. So one can put $x_{i, j}^{t}=0$ if $i$ or $j$ is odd.

The method gives, in the range $n \leq 28$, the new upper bounds on $A(n, d)$ given in Table I (cf. the tables given by Best, Brouwer, MacWilliams, Odlyzko, and Sloane [3] and Agrell, Vardy, and Zeger [2]; $A(25,8) \leq 5557$ and $A(26,10) \leq 989$ were shown by Mounits, Etzion, and Litsyn [11], $A(22,10) \geq 64$ by Östergård [12], and $A(25,10) \geq 192$ and $A(26,10) \geq 384$ by Elssel and Zimmermann [5] (see also Andries Brouwer's website http://www.win.tue.nl/ aeb/codes/ binary-1.html)).

\begin{tabular}{|r|r|r|r|r|r|}
\hline$n$ & $d$ & $\begin{array}{r}\text { best } \\
\text { lower } \\
\text { bound } \\
\text { known }\end{array}$ & $\begin{array}{r}\text { new } \\
\text { upper } \\
\text { bound }\end{array}$ & $\begin{array}{c}\text { best upper } \\
\text { bound } \\
\text { previously } \\
\text { known }\end{array}$ & $\begin{array}{c}\text { Delsarte } \\
\text { bound }\end{array}$ \\
\hline 19 & 6 & 1024 & 1280 & 1288 & 1289 \\
23 & 6 & 8192 & 13766 & 13774 & 13775 \\
25 & 6 & 16384 & 47998 & 48148 & 48148 \\
\hline 19 & 8 & 128 & 142 & 144 & 145 \\
20 & 8 & 256 & 274 & 279 & 290 \\
25 & 8 & 4096 & 5477 & 5557 & 6474 \\
27 & 8 & 8192 & 17768 & 17804 & 18189 \\
28 & 8 & 16384 & 32151 & 32204 & 32206 \\
\hline 22 & 10 & 64 & 87 & 88 & 95 \\
25 & 10 & 192 & 503 & 549 & 551 \\
26 & 10 & 384 & 886 & 989 & 1040 \\
\hline
\end{tabular}

TABLE I

NEW UPPER BOUNDS ON $A(n, d)$

Our computations were done by the algorithm SDPT3 version 3.02 (cf. Tütüncü, Toh, and Todd [16]), which is available through the web on the NEOS Server for Optimization (http://www-neos.mcs.anl.gov/neos/server-solvers. html\#SDP). The answers have been confirmed by the algorithm DSDP version 5.5, available on the same server.

We note that the new bound is stronger than the Delsarte bound, which is equal to the maximum value of $\sum_{i}\left(\begin{array}{l}n \\ i\end{array}\right) x_{i, 0}^{0}$ subject to the condition that $x_{i, 0}^{0} \geq 0$ for all $i$ and $x_{i, 0}^{0}=0$ if $1 \leq i \leq d-1$, and to the condition that

$$
\sum_{i, j, t} x_{i+j-2 t, 0}^{0} M_{i, j}^{t} \text { is positive semidefinite. }
$$

(This matrix belongs to the Bose-Mesner algebra, which is a 
subalgebra of $\mathcal{A}_{n}$.) The latter condition is equivalent to the Delsarte inequalities (involving the Krawtchouk polynomial). (Then the variables different from the $x_{i, 0}^{0}$ are superfluous and can be deleted.) Condition (23) is a consequence of the positive semidefiniteness of the matrices

$$
\sum_{i, j, t} x_{i, j}^{t} M_{i, j}^{t} \text { and } \sum_{i, j, t}\left(x_{i+j-2 t, 0}^{0}-x_{i, j}^{t}\right) M_{i, j}^{t},
$$

which is, as we saw, equivalent to (19).

A sharpening of the bound can be obtained by adding the conditions (for appropriate $i$ )

$$
\left(\begin{array}{l}
n \\
i
\end{array}\right) x_{i, 0}^{0} \leq A^{*}(n, d, i)
$$

where $A^{*}(n, d, i)$ is any upper bound on the maximum size of a constant-weight code of word length $n$, minimum distance at least $d$, and constant weight $i$. Adding these constraints to the new bound seems less effective than adding them to the Delsarte bound, as the new bound implicitly contains the Delsarte bound for the Johnson schemes. Using known upper bounds $A^{*}(n, d, i)$, we did not obtain in this way any improvement in the above table.

\section{Some background}

Above we have introduced the Terwilliger algebra of the Hamming cube in a way that is convenient for our purposes, which differs slightly from the usual (but equivalent) definition. In the usual terminology, we consider the Terwilliger algebra $T=T(\mathbf{0})$ of the Hamming cube $H(n, 2)$ with respect to $\mathbf{0}$. This is the algebra generated by the $\mathcal{P} \times \mathcal{P} 0,1$ matrices $A_{d}$ and $E_{d}^{*}$ for $d=0, \ldots, n$, where $\left(A_{d}\right)_{X, Y}=1 \Longleftrightarrow$ $|X \triangle Y|=d$, and $\left(E_{d}^{*}\right)_{X, Y}=1 \Longleftrightarrow X=Y$ and $|X|=d$. Then $M_{i, j}^{t}=E_{i}^{*} A_{i+j-2 t} E_{j}^{*}$ for all $i, j, t$. Conversely, $A_{d}=$ $\sum_{i, j, t ; i+j-2 t=d} M_{i, j}^{t}$ and $E_{d}^{*}=M_{d, d}^{d}$ for each $d$. So $T(\mathbf{0})$ coincides with our algebra $\mathcal{A}_{n}$.

Basic properties of the Terwilliger algebra of the Hamming cube were found by Go [6]. In particular, Go identified the irreducible $T$-modules of the algebra, which implies the block sizes and block multiplicities of $\mathcal{A}_{n}$. Go also described bases for these modules. Our paper needs, and gives, a more explicit description of these bases. It also yields an explicit decomposition of the Terwilliger algebra into irreducible constituents.

The present research roots in two basic papers presenting eigenvalue techniques to obtain upper bounds: Delsarte [4], giving a bound on codes based on association schemes, and Lovász [7], giving a bound on the Shannon capacity of a graph. It was shown by McEliece, Rodemich, and Rumsey [10] and Schrijver [13] that the Delsarte bound is a special case of (a close variant of) the Lovász bound. (This is not to say that the Lovász bound supersedes the Delsarte bound: essential in the latter bound is a reduction of the $2^{n}$-vertex graph problem to a linear programming problem of order $n$.) An extension of the Lovász bound based on 'matrix cuts' was given by Lovász and Schrijver [8]. Applying a variant of matrix cuts to the coding problem leads to considering the Terwilliger algebra as above.

\section{BLOCK-DIAGONALISATION OF THE TERWILLIGER ALGEBRA}

In this section we show that (8) indeed describes the blockdiagonalisation of $\mathcal{A}_{n}$. For $k=0, \ldots,\left\lfloor\frac{n}{2}\right\rfloor$, let $L_{k}$ be the linear space

$$
\begin{aligned}
& L_{k}:=\left\{b \in \mathbb{R}^{\mathcal{P}} \mid M_{k-1, k}^{k-1} b=0, \text { and } b_{X}=0 \text { if }|X| \neq\right. \\
& k\} .
\end{aligned}
$$

Then

$$
M_{i, k}^{i} b=0 \text { for all } i<k \text { and } b \in L_{k},
$$

since $M_{i, k-1}^{i} M_{k-1, k}^{k-1}=(k-i) M_{i, k}^{i}$.

The dimension of $L_{k}$ is given by:

$$
\operatorname{dim} L_{k}=\left(\begin{array}{l}
n \\
k
\end{array}\right)-\left(\begin{array}{c}
n \\
k-1
\end{array}\right),
$$

since:

Proposition 2: For each $k \leq\left\lfloor\frac{n}{2}\right\rfloor, M_{k-1, k}^{k-1}$ has rank $\left(\begin{array}{c}n \\ k-1\end{array}\right)$.

Proof: We have

$$
\begin{aligned}
& M_{k-1, k}^{k-1} M_{k, k-1}^{k-1}= \\
& M_{k-1, k-2}^{k-2} M_{k-2, k-1}^{k-2}+(n-2 k+2) M_{k-1, k-1}^{k-1} .
\end{aligned}
$$

As $M_{k-1, k-2}^{k-2} M_{k-2, k-1}^{k-2}$ is positive semidefinite, as $n-2 k+$ $2>0$, and as $M_{k-1, k-1}^{k-1}$ is positive semidefinite of rank $\left(\begin{array}{c}n \\ k-1\end{array}\right)$, we know that $M_{k-1, k}^{k-1} M_{k, k-1}^{k-1}$ has rank $\left(\begin{array}{c}n \\ k-1\end{array}\right)$. Hence also $M_{k-1, k}^{k-1}$ has rank $\left(\begin{array}{c}n \\ k-1\end{array}\right)$.

The following formula is basic to our results (note that $c^{\top} b=0$ if $c \in L_{l}, b \in L_{k}$, and $l \neq k$ ):

Proposition 3: For $i, j, k, l, t \in\{0, \ldots, n\}$ with $k, l \leq\left\lfloor\frac{n}{2}\right\rfloor$, and for $c \in L_{l}, b \in L_{k}$ :

$$
c^{\top} M_{l, i}^{l} M_{i, j}^{t} M_{j, k}^{k} b=\beta_{i, j, k}^{t} c^{\top} b .
$$

Proof: First we have for each $s \in\{0, \ldots, n\}$ :

$$
M_{l, s}^{s} M_{s, k}^{s}=\sum_{p=0}^{n}\left(\begin{array}{l}
p \\
s
\end{array}\right) M_{l, k}^{p},
$$

since the entry of this matrix in position $(X, Y)$, with $|X|=l$ and $|Y|=k$, is equal to the number of common subsets of $X$ and $Y$ of size $s$.

Equation (31) implies for all $l, k, p \in\{0, \ldots, n\}$ :

$$
M_{l, k}^{p}=\sum_{s=0}^{n}(-1)^{s-p}\left(\begin{array}{l}
s \\
p
\end{array}\right) M_{l, s}^{s} M_{s, k}^{s},
$$

since 
(33)

$$
\begin{aligned}
& \sum_{s=0}^{n}(-1)^{s-p}\left(\begin{array}{l}
s \\
p
\end{array}\right) M_{l, s}^{s} M_{s, k}^{s}= \\
& \sum_{s=0}^{n}(-1)^{s-p}\left(\begin{array}{l}
s \\
p
\end{array}\right) \sum_{t=0}^{n}\left(\begin{array}{l}
t \\
s
\end{array}\right) M_{l, k}^{t}= \\
& \sum_{t=0}^{n} \sum_{s=0}^{n}(-1)^{s-p}\left(\begin{array}{l}
s \\
p
\end{array}\right)\left(\begin{array}{l}
t \\
s
\end{array}\right) M_{l, k}^{t}=\sum_{t=0}^{n} \delta_{t, p} M_{l, k}^{t}=M_{l, k}^{p},
\end{aligned}
$$

where $\delta_{t, p}=1$ if $t=p$, and $\delta_{t, p}=0$ else.

Equation (32) implies that for all $l, k, p \in\{0, \ldots, n\}$ and $b \in L_{k}$ :

$$
M_{l, k}^{p} b=(-1)^{k-p}\left(\begin{array}{l}
k \\
p
\end{array}\right) M_{l, k}^{k} b,
$$

since $M_{l, s}^{s} M_{s, k}^{s} b=0$ if $s \neq k$ : if $s<k$ then $M_{s, k}^{s} b=0$ (by (27)) and if $s>k$ then $M_{s, k}^{s}=\mathbf{0}$.

Equation (34) implies

$$
M_{p, j}^{p} M_{j, k}^{k} b=\left(\begin{array}{c}
n-k-p \\
j-p
\end{array}\right) M_{p, k}^{k} b
$$

since

$$
\begin{aligned}
& M_{p, j}^{p} M_{j, k}^{k} b=\sum_{t=0}^{n}\left(\begin{array}{c}
n-p-k+t \\
n-j
\end{array}\right) M_{p, k}^{t} b= \\
& \sum_{t=0}^{n}\left(\begin{array}{c}
n-p-k+t \\
n-j
\end{array}\right)(-1)^{k-t}\left(\begin{array}{c}
k \\
t
\end{array}\right) M_{p, k}^{k} b=\left(\begin{array}{c}
n-k-p \\
j-p
\end{array}\right) M_{p, k}^{k} b .
\end{aligned}
$$

We finally obtain (30) (using (32) and three times (35)):

$$
\begin{aligned}
& c^{\top} M_{l, i}^{l} M_{i, j}^{t} M_{j, k}^{k} b= \\
& \sum_{p=0}^{n}(-1)^{t-p}\left(\begin{array}{c}
p \\
t
\end{array}\right) c^{\top} M_{l, i}^{l} M_{i, p}^{p} M_{p, j}^{p} M_{j, k}^{k} b= \\
& \sum_{p=0}^{n}(-1)^{t-p}\left(\begin{array}{c}
p \\
t
\end{array}\right)\left(\begin{array}{c}
n-l-p \\
i-p
\end{array}\right)\left(\begin{array}{c}
n-k-p \\
j-p
\end{array}\right) c^{\top} M_{l, p}^{l} M_{p, k}^{k} b= \\
& \sum_{p=0}^{n}(-1)^{t-p}\left(\begin{array}{c}
p \\
t
\end{array}\right)\left(\begin{array}{c}
n-l-p \\
i-p
\end{array}\right)\left(\begin{array}{c}
n-k-p \\
j-p
\end{array}\right)\left(\begin{array}{c}
n-l-k \\
n-p-k
\end{array}\right) c^{\top} M_{l, k}^{k} b .
\end{aligned}
$$

By (27), the latter expression is nonzero only if $l=k$, in which case it is equal to $\beta_{i, j, k}^{t} c^{\top} b$. Since $c^{\top} b=0$ if $l \neq k$, this proves the proposition.

This implies:

Proposition 4: For $i, j, k, l \in\{0, \ldots, n\}$ with $k, l \leq\left\lfloor\frac{n}{2}\right\rfloor$, and for $c \in L_{l}, b \in L_{k}$ :

$$
c^{\top} M_{l, i}^{l} M_{j, k}^{k} b= \begin{cases}\left(\begin{array}{c}
n-2 k \\
i-k
\end{array}\right) c^{\top} b & \text { if } l=k, i=j, \\
0 & \text { otherwise }\end{cases}
$$

Proof: Since $M_{l, i}^{l} M_{j, k}^{k}=\mathbf{0}$ if $i \neq j$, we can assume $i=j$. Then

$$
c^{\top} M_{l, i}^{l} M_{i, k}^{k} b=c^{\top} M_{l, i}^{l} M_{i, i}^{i} M_{i, k}^{k} b
$$

If $l \neq k$, this is 0 by (30). If $l=k$, then, again by (30), it is equal to $\beta_{i, i, k}^{i} c^{\top} b=\left(\begin{array}{c}n-2 k \\ i-k\end{array}\right) c^{\top} b$.

For each $k=0, \ldots,\left\lfloor\frac{n}{2}\right\rfloor$, choose an orthonormal basis $\mathcal{B}_{k}$ of $L_{k}$. By (28), $\left|\mathcal{B}_{k}\right|=\left(\begin{array}{l}n \\ k\end{array}\right)-\left(\begin{array}{c}n \\ k-1\end{array}\right)$. Let

$$
\begin{aligned}
& V:=\left\{(k, b, i) \mid k \in\left\{0, \ldots,\left\lfloor\frac{n}{2}\right\rfloor\right\}, b \in \mathcal{B}_{k}, i \in\right. \\
& \{k, k+1, \ldots, n-k\}\}
\end{aligned}
$$

Then

(41) $|V|=2^{n}$

since

$$
\begin{aligned}
& |V|=\sum_{i=0}^{n} \sum_{k=0}^{\min \{i, n-i\}}\left(\left(\begin{array}{l}
n \\
k
\end{array}\right)-\left(\begin{array}{c}
n \\
k-1
\end{array}\right)\right)= \\
& \sum_{i=0}^{n}\left(\begin{array}{c}
n \\
\min \{i, n-i\}
\end{array}\right)=\sum_{i=0}^{n}\left(\begin{array}{l}
n \\
i
\end{array}\right)=2^{n} .
\end{aligned}
$$

For each $(k, b, i) \in V$, define $u_{k, b, i} \in \mathbb{R}^{\mathcal{P}}$ by

$$
u_{k, b, i}:=\left(\begin{array}{c}
n-2 k \\
i-k
\end{array}\right)^{-\frac{1}{2}} M_{i, k}^{k} b .
$$

With (41), Proposition 4 implies that the $u_{k, b, i}$ 's form an orthonormal basis for $\mathbb{R}^{\mathcal{P}}$. Let $U$ be the $\mathcal{P} \times V$ matrix whose $(k, b, i)$-th column equals $u_{k, b, i}$, for $(k, b, i) \in V$. Then for each triple $i, j, t$, the matrix $\bar{M}_{i, j}^{t}:=U^{\top} M_{i, j}^{t} U$ is in blockdiagonal form. This will follow from:

Proposition 5: For $\left(l, c, i^{\prime}\right),\left(k, b, j^{\prime}\right) \in V$ and $i, j, t \in$ $\{0, \ldots, n\}$

$$
\begin{aligned}
& \left(\widetilde{M}_{i, j}^{t}\right)_{\left(l, c, i^{\prime}\right),\left(k, b, j^{\prime}\right)}= \\
& \begin{cases}\left(\begin{array}{c}
n-2 k \\
i-k
\end{array}\right)^{-\frac{1}{2}}\left(\begin{array}{c}
n-2 k \\
j-k
\end{array}\right)^{-\frac{1}{2}} \beta_{i, j, k}^{t} & \text { if } l=k, i=i^{\prime}, j=j^{\prime}, \\
0 & \text { and } b=c, \\
0 & \text { otherwise. }\end{cases}
\end{aligned}
$$

Proof: We have

$$
\begin{aligned}
& \left(\widetilde{M}_{i, j}^{t}\right)_{\left(l, c, i^{\prime}\right),\left(k, b, j^{\prime}\right)}=u_{l, c, i^{\prime}}^{\top} M_{i, j}^{t} u_{k, b, j^{\prime}}= \\
& \left(\begin{array}{c}
n-2 l \\
i^{\prime}-l
\end{array}\right)^{-\frac{1}{2}}\left(\begin{array}{c}
n-2 k \\
j^{\prime}-k
\end{array}\right)^{-\frac{1}{2}} c^{\top} M_{l, i^{\prime}}^{l} M_{i, j}^{t} M_{j^{\prime}, k}^{k} b .
\end{aligned}
$$

This is 0 if $i^{\prime} \neq i$ or $j^{\prime} \neq j$. So we can assume that $i=i^{\prime}$ and $j=j^{\prime}$. Then (30) and (45) imply (44).

This implies that each matrix in $U^{\top} \mathcal{A}_{n} U$ is a block-diagonal matrix determined by the partition of $V$ into the classes

$$
V_{k, b}:=\{(k, b, i) \mid k \leq i \leq n-k\}
$$

for $k=0, \ldots,\left\lfloor\frac{n}{2}\right\rfloor$ and $b \in \mathcal{B}_{k}$. Indeed, if $\left(l, c, i^{\prime}\right),\left(k, b, j^{\prime}\right) \in$ $V$ then $\left(\widetilde{M}_{i, j}^{t}\right)_{\left(l, c, i^{\prime}\right),\left(k, b, j^{\prime}\right)}=0$ if $l \neq k$ or $c \neq b$. 
Moreover, for $k \in\left\{0, \ldots,\left\lfloor\frac{n}{2}\right\rfloor\right\}, b, c \in \mathcal{B}_{k}$, and $i^{\prime}, j^{\prime} \in$ $\{k, \ldots, n-k\}$ we have by (44)

$$
\left(\widetilde{M}_{i, j}^{t}\right)_{\left(k, b, i^{\prime}\right),\left(k, b, j^{\prime}\right)}=\left(\widetilde{M}_{i, j}^{t}\right)_{\left(k, c, i^{\prime}\right),\left(k, c, j^{\prime}\right)} .
$$

So for each fixed $k$, the blocks determined by the $V_{k, b}$ (over $b \in \mathcal{B}_{k}$ ) are equal.

For each $k$ and each $b \in \mathcal{B}_{k}$, the block determined by $V_{k, b}$ has size $\left|V_{k, b}\right|=n-2 k+1$. Now

$$
\sum_{k=0}^{\left\lfloor\frac{n}{2}\right\rfloor}(n-2 k+1)^{2}=\left(\begin{array}{c}
n+3 \\
3
\end{array}\right)
$$

(Proof: Induction on $n$. It is true for $n=0$ and $n=1$. Moreover, $\left(\begin{array}{c}n+3 \\ 3\end{array}\right)-\left(\begin{array}{c}n+1 \\ 3\end{array}\right)=\frac{1}{6}((n+3)(n+2)(n+1)-$ $(n+1) n(n-1))=\frac{1}{6}\left(\left(n^{3}+6 n^{2}+11 n+6\right)-\left(n^{3}-n\right)\right)=$ $\frac{1}{6}\left(6 n^{2}+12 n+6\right)=(n+1)^{2}$. $)$

As $\left(\begin{array}{c}n+3 \\ 3\end{array}\right)$ is the dimension of $\mathcal{A}_{n}$ (by (3)), we can conclude that $\mathcal{A}_{n}$ is (as an algebra) isomorphic to the direct sum

$$
\bigoplus_{k=0}^{\left\lfloor\frac{n}{2}\right\rfloor} \mathbb{C}^{V_{k}, b_{k} \times V_{k}, b_{k}}
$$

where $b_{k}$ is an arbitrary element of $\mathcal{B}_{k}$.

In other words, define, for each $k=0, \ldots,\left\lfloor\frac{n}{2}\right\rfloor$,

$$
N_{k}:=\{k, k+1, \ldots, n-k\}
$$

Then the $k$ th block $B_{k}$ belongs to $\mathbb{C}^{N_{k} \times N_{k}}$, and (using (44)):

Theorem 1: $\mathcal{A}_{n}$ is isomorphic to $\bigoplus_{k=0}^{\left\lfloor\frac{n}{2}\right\rfloor} \mathbb{C}^{N_{k} \times N_{k}}$, where (2) maps in $\mathbb{C}^{N_{k} \times N_{k}}$ to matrix

$$
\left(\sum_{t}\left(\begin{array}{c}
n-2 k \\
i-k
\end{array}\right)^{-\frac{1}{2}}\left(\begin{array}{c}
n-2 k \\
j-k
\end{array}\right)^{-\frac{1}{2}} \beta_{i, j, k}^{t} x_{i, j}^{t}\right)_{i, j=k}^{n-k}
$$

\section{CONSTANT-WEIGHT CODES}

We now go over to derive a similar bound for constantweight codes, which is based on considering a tensor product of the algebra $\mathcal{A}_{n}$. In the previous sections we fixed $n$, but now it will be convenient to have $n$ as parameter in our notation. Therefore, we will denote the objects $\mathcal{P}, M_{i, j}^{t}, B_{k}, \beta_{i, j, k}^{t}$, and $U$ by $\mathcal{P}_{n}, M_{i, j}^{t, n}, B_{k}^{n}, \beta_{i, j, k}^{t, n}$, and $U_{n}$, respectively.

\section{A. The algebras $\mathcal{A}_{w, v}$ and $\mathcal{B}_{w, v}$}

Choose $n$ and $w$ with $w \leq n$, and define $v:=n-w$. Let $\mathcal{A}_{w, v}$ be the $\mathrm{C} *$-algebra generated by the tensor products ${ }^{1}$ of matrices in $\mathcal{A}_{w}$ and $\mathcal{A}_{v}$. So $\mathcal{A}_{w, v}$ is equal to the set of matrices

\footnotetext{
${ }^{1}$ The tensor product of an $A \times B$ matrix $M$ and a $C \times D$ matrix $N$ is the $(A \times C) \times(B \times D)$ matrix $M \circ N$ given by $(M \circ N)_{(a, c),(b, d)}:=M_{a, b} N_{c, d}$ for $(a, c) \in A \times C$ and $(b, d) \in B \times D$.
}

$$
\sum_{i, j, t, i^{\prime}, j^{\prime}, s} z_{i, j, i^{\prime}, j^{\prime}}^{t, s} M_{i, j}^{t, w} \circ M_{i^{\prime}, j^{\prime}}^{s, v}
$$

with $z_{i, j, i^{\prime}, j^{\prime}}^{t, s} \in \mathbb{C}$. by

The algebra $\mathcal{A}_{w, v}$ can be brought into block-diagonal form

$$
\left(U_{w} \circ U_{v}\right)^{\top} \mathcal{A}_{w, v}\left(U_{w} \circ U_{v}\right)
$$

since

$$
\begin{aligned}
& \left(U_{w} \circ U_{v}\right)^{\top}\left(M_{i, j}^{t, w} \circ M_{i^{\prime}, j^{\prime}}^{s, v}\right)\left(U_{w} \circ U_{v}\right)= \\
& \left(U_{w}^{\top} M_{i, j}^{t, w} U_{w}\right) \circ\left(U_{v}^{\top} M_{i^{\prime}, j^{\prime}}^{s, v} U_{v}\right)
\end{aligned}
$$

for all $i, j, i^{\prime}, j^{\prime}, t, s$. Then the blocks of $\mathcal{A}_{w, v}$ are spanned by the tensor products $B_{k}^{w} \circ B_{l}^{v}$ of a block $B_{k}^{w}$ of $\mathcal{A}_{w}$ and a block $B_{l}^{v}$ of $\mathcal{A}_{v}$. Note that $B_{k}^{w} \circ B_{l}^{v}$ is a $\left(W_{k} \times V_{l}\right) \times\left(W_{k} \times V_{l}\right)$ matrix, where we denote

$$
\begin{aligned}
& W_{k}:=\{k, k+1, \ldots, w-k\} \text { and } \\
& V_{l}:=\{l, l+1, \ldots, v-l\}
\end{aligned}
$$

By Theorem 1 and by the definition of tensor product, matrix (52) maps in $B_{k}^{w} \circ B_{l}^{v}$ to the matrix

$$
\begin{aligned}
& \left(\sum_{t, s}\left(\begin{array}{c}
w-2 k \\
i-k
\end{array}\right)^{-\frac{1}{2}}\left(\begin{array}{c}
w-2 k \\
j-k
\end{array}\right)^{-\frac{1}{2}}\left(\begin{array}{c}
v-2 l \\
i^{\prime}-l
\end{array}\right)^{-\frac{1}{2}}\left(\begin{array}{c}
v-2 l \\
j^{\prime}-l
\end{array}\right)^{-\frac{1}{2}} .\right. \\
& \left.\cdot \beta_{i, j, k}^{t, w} \beta_{i^{\prime}, j^{\prime}, l}^{s, v} z_{i, j, i^{\prime}, j^{\prime}}^{t, s}\right)_{\left(i, i^{\prime}\right),\left(j, j^{\prime}\right) \in W_{k} \times V_{l}}
\end{aligned}
$$

We next consider the subalgebra $\mathcal{B}_{w, v}$ of $\mathcal{A}_{w, v}$ consisting of all matrices

$$
\sum_{i, j, t, s} y_{i, j}^{t, s} M_{i, j}^{t, w} \circ M_{i, j}^{s, v}
$$

with $y_{i, j}^{t, s} \in \mathbb{C}$. So $\mathcal{B}_{w, v}$ consists of all matrices (52) with $z_{i, j, i^{\prime}, j^{\prime}}^{t, s}=0$ if $i \neq i^{\prime}$ or $j \neq j^{\prime}$.

The image (56) of (57) in block $B_{k}^{w} \circ B_{l}^{v}$ has zeros in positions $\left(i, i^{\prime}\right),\left(j, j^{\prime}\right)$ with $i \neq i^{\prime}$ or $j \neq j^{\prime}$. Deleting these rows and columns, we obtain a block of order $\left|W_{k} \cap V_{l}\right|$ (of zero order if $W_{k} \cap V_{l}=\emptyset$ ). Then (57) maps in this block to

$$
\begin{aligned}
& \left(\sum_{t, s}\left(\begin{array}{c}
w-2 k \\
i-k
\end{array}\right)^{-\frac{1}{2}}\left(\begin{array}{c}
w-2 k \\
j-k
\end{array}\right)^{-\frac{1}{2}}\left(\begin{array}{c}
v-2 l \\
i-l
\end{array}\right)^{-\frac{1}{2}}\left(\begin{array}{c}
v-2 l \\
j-l
\end{array}\right)^{-\frac{1}{2}} .\right. \\
& \left.\cdot \beta_{i, j, k}^{t, w} \beta_{i, j, l}^{s, v} y_{i, j}^{t, s}\right)_{i, j \in W_{k} \cap V_{l}}
\end{aligned}
$$

where we have identified any $i \in W_{k} \cap V_{l}$ with the pair $(i, i) \in$ $W_{k} \times V_{l}$.

This in fact gives the block-diagonalisation of $\mathcal{B}_{w, v}$. For consider any complex $\left(W_{k} \cap V_{l}\right) \times\left(W_{k} \cap V_{l}\right)$ matrix $L$. Extend $L$ by zeros so as to obtain a $\left(W_{k} \times V_{l}\right) \times\left(W_{k} \times V_{l}\right)$ matrix $L^{\prime}$. As (56) gives the block-diagonalisation of $\mathcal{A}_{w, v}$, we know that $L^{\prime}$ is equal to (56) for some $z_{i, j, i^{\prime}, j^{\prime}}^{t, s}$. Resetting $z_{i, j, i^{\prime}, j^{\prime}}^{t, s}$ 
to 0 if $i \neq i^{\prime}$ or $j \neq j^{\prime}$ does not change $L^{\prime}$. Hence $L$ can be given as (58), for $y_{i, j}^{t, s}:=z_{i, j, i, j}^{t, s}$.

Incidentally, this implies

$$
\operatorname{dim}\left(\mathcal{B}_{w, v}\right)=\sum_{k=0}^{\left\lfloor\frac{w}{2}\right\rfloor} \sum_{l=0}^{\left\lfloor\frac{v}{2}\right\rfloor}\left|W_{k} \cap V_{l}\right|^{2}
$$

\section{B. Application to constant-weight coding}

We proceed as in Section I. Let $C \subseteq \mathcal{P}_{n}$ be any constantweight code of word length $n$ and constant weight $w$. Fix a set $X \in \mathcal{P}_{n}$ with $|X|=w$. We will identify $\mathcal{P}_{n}$ and $\mathcal{P}_{w} \times \mathcal{P}_{v}$, by identifying any $Y \in \mathcal{P}_{n}$ with the pair $(X \backslash Y, Y \backslash X) \in$ $\mathcal{P}_{w} \times \mathcal{P}_{v}$.

Let $\Pi$ be the set of (distance-preserving) automorphisms $\pi$ of $\mathcal{P}_{n}$ fixing $\emptyset$ and with $X \in \pi(C)$, and let $\Pi^{\prime}$ be the set of automorphisms $\pi$ of $\mathcal{P}_{n}$ fixing $\emptyset$ and with $X \notin \pi(C)$. Define the matrices $R$ and $R^{\prime}$ by:

$$
\begin{aligned}
& R:=\sum_{\pi \in \Pi}|\Pi|^{-1} \chi^{\pi(C)}\left(\chi^{\pi(C)}\right)^{\top} \text { and } \\
& R^{\prime}:=\sum_{\pi \in \Pi^{\prime}}\left|\Pi^{\prime}\right|^{-1} \chi^{\pi(C)}\left(\chi^{\pi(C)}\right)^{\top}
\end{aligned}
$$

Again, as $R$ and $R^{\prime}$ are sums of positive semidefinite matrices, they are positive semidefinite. Moreover, $R$ and $R^{\prime}$ belong to $\mathcal{B}_{w, v}$, using the identification of $\mathcal{P}_{n}$ and $\mathcal{P}_{w} \times \mathcal{P}_{v}$ :

$$
\begin{aligned}
& R=\sum_{i, j, t, s} y_{i, j}^{t, s} M_{i, j}^{t, w} \circ M_{i, j}^{s, v} \text { and } \\
& R^{\prime}=\frac{|C|}{2^{n}-|C|} \sum_{i, j, t, s}\left(y_{i+j-t-s, 0}^{0,0}-y_{i, j}^{t, s}\right) M_{i, j}^{t, w} \circ M_{i, j}^{s, v},
\end{aligned}
$$

with

$$
y_{i, j}^{t, s}:=\frac{1}{|C|\left(_{i-t, j-t, t}\right)\left(\begin{array}{c}
v \\
i-s, j-s, s
\end{array}\right)} \mu_{i, j}^{t, s},
$$

where

$$
\begin{aligned}
& \mu_{i, j}^{t, s}:=\text { the number of triples }(X, Y, Z) \in C^{3} \text { with } \\
& |X \backslash Y|=i,|X \backslash Z|=j,|(X \backslash Y) \cap(X \backslash Z)|=t,
\end{aligned}
$$
and $|(Y \backslash X) \cap(Z \backslash X)|=s$.

The equations in (61) can be proved similarly as Proposition 1.

The positive semidefiniteness of $R$ and $R^{\prime}$ is by (58) equivalent to:

(64) for each $k=0, \ldots,\left\lfloor\frac{w}{2}\right\rfloor$ and $l=0, \ldots,\left\lfloor\frac{v}{2}\right\rfloor$, the matrices

$$
\left(\sum_{t, s} \beta_{i, j, k}^{t, w} \beta_{i, j, l}^{s, v} y_{i, j}^{t, s}\right)_{i, j \in W_{k} \cap V_{l}}
$$

and

$$
\left(\sum_{t, s} \beta_{i, j, k}^{t, w} \beta_{i, j, l}^{s, v}\left(y_{i+j-t-s, 0}^{0,0}-y_{i, j}^{t, s}\right)\right)_{i, j \in W_{k} \cap V_{l}}
$$

are positive semidefinite.

The $y_{i, j}^{t, s}$, s moreover satisfy the following constraints, where (iv) holds if $C$ has minimum distance at least $d$ :

(i) $y_{0,0}^{0,0}=1$,

(ii) $0 \leq y_{i, j}^{t, s} \leq y_{i, 0}^{0,0}$ and $y_{i, 0}^{0,0}+y_{j, 0}^{0,0} \leq 1+y_{i, j}^{t, s}$ for all $i, j, t, s \in\{0, \ldots, \min \{w, v\}\}$

(iii) $y_{i, j}^{t, s}=y_{i^{\prime}, j^{\prime}}^{t^{\prime}}, s^{\prime}$ if $t^{\prime}-s^{\prime}=t-s$ and $\left(i^{\prime}, j^{\prime}, i^{\prime}+j^{\prime}-\right.$ $\left.t^{\prime}-s^{\prime}\right)$ is a permutation of $(i, j, i+j-t-s)$,

(iv) $y_{i, j}^{t, s}=0$ if $\{2 i, 2 j, 2(i+j-t-s)\} \cap\{1, \ldots, d-$ $1\} \neq \emptyset$.

(Condition (ii) follows from the fact that each row of $R$ and $R^{\prime}$ is nonnegative and is dominated by its diagonal entry (by (60)). Conditions (iii) and (iv) follow from the fact that $\mu_{i, j}^{t, s}$ is equal to the number of triples $(X, Y, Z)$ in $C^{3}$ with $|X \triangle Y|=2 i$, $|X \triangle Z|=2 j,|Y \triangle Z|=2(i+j-t-s)$, and $|X \triangle Y \triangle Z|=$ $w+2 t-2 s$, as follows directly from (63).)

Now

$$
|C|=\sum_{i=0}^{\min \{w, v\}}\left(\begin{array}{c}
w \\
i
\end{array}\right)\left(\begin{array}{c}
v \\
i
\end{array}\right) y_{i, 0}^{0,0}
$$

since $|C|^{2}=\sum_{i=0}^{\min \{w, v\}} \mu_{i, 0}^{0,0}$. Hence we obtain an upper bound on $A(n, d, w)$ by considering the $y_{i, j}^{t, s}$ as variables, and by

$$
\begin{aligned}
& \text { maximizing } \sum_{i=0}^{\min \{w, v\}}\left(\begin{array}{c}
w \\
i
\end{array}\right)\left(\begin{array}{c}
v \\
i
\end{array}\right) y_{i, 0}^{0,0} \text { subject to conditions } \\
& \text { (64) and (65). }
\end{aligned}
$$

This is a semidefinite programming problem with $O\left(w^{4}\right)$ variables, and it can be solved in time polynomial in $n$. In the range $n \leq 28$, it gives the new bounds given in Table II (cf. the tables given by Best, Brouwer, MacWilliams, Odlyzko, and Sloane [3] and Agrell, Vardy, and Zeger [1], and Erik Agrell's website http://www.s2.chalmers.se/ $\sim$ agrell/bounds/cw.html). Note that it implies the exact value $A(23,8,11)=1288$.

Again, this new bound strengthens the Delsarte bound for constant-weight codes, as can be seen by an argument similar to that given in Section I.

Acknowledgements. I thank Rob Ellis, Dion Gijswijt, Monique Laurent, and Dima Pasechnik for very helpful discussions and comments. I am moreover grateful to the two referees and to the editor, Khaled Abdel-Ghaffar, for useful suggestions as to the presentation of the results. 


\begin{tabular}{|c|c|c|c|c|c|c|}
\hline$n$ & $d$ & $w$ & $\begin{array}{l}\text { best } \\
\text { lower } \\
\text { bound } \\
\text { known }\end{array}$ & $\begin{array}{c}\text { new } \\
\text { upper } \\
\text { bound }\end{array}$ & $\begin{array}{l}\text { best upper } \\
\text { bound } \\
\text { previously } \\
\text { known }\end{array}$ & $\begin{array}{c}\text { Delsarte } \\
\text { bound }\end{array}$ \\
\hline 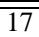 & $\overline{\overline{6}}$ & $\overline{77}$ & 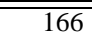 & 228 & 234 & 249 \\
\hline 17 & 6 & 8 & 184 & 280 & 283 & 283 \\
\hline 18 & 6 & 6 & 132 & 199 & 202 & 204 \\
\hline 19 & 6 & 8 & 408 & 718 & 734 & 751 \\
\hline 21 & 6 & 9 & 1184 & 2359 & 2364 & 2364 \\
\hline 21 & 6 & 10 & 1454 & 2685 & 2702 & 2702 \\
\hline 22 & 6 & 9 & 1792 & 3736 & 3775 & 3775 \\
\hline 22 & 6 & 10 & 2182 & 4415 & 4416 & 4734 \\
\hline 26 & 6 & 11 & 12037 & 42075 & 42081 & 42081 \\
\hline 26 & 6 & 12 & 14836 & 50169 & 50204 & 52440 \\
\hline 21 & 8 & 9 & 280 & 314 & 320 & 358 \\
\hline 21 & 8 & 10 & 336 & 383 & 399 & 464 \\
\hline 22 & 8 & 9 & 280 & 473 & 493 & 597 \\
\hline 22 & 8 & 10 & 616 & 634 & 641 & 758 \\
\hline 22 & 8 & 11 & 672 & 680 & 766 & 805 \\
\hline 23 & 8 & 9 & 400 & 707 & 796 & 830 \\
\hline 23 & 8 & 10 & 616 & 1025 & 1109 & 1111 \\
\hline 23 & 8 & 11 & 1288 & 1288 & 1328 & 1417 \\
\hline 24 & 8 & 9 & 640 & 1041 & 1143 & 1160 \\
\hline 24 & 8 & 10 & 960 & 1551 & 1639 & 1639 \\
\hline 24 & 8 & 11 & 1288 & 2142 & 2188 & 2305 \\
\hline 25 & 8 & 9 & 829 & 1486 & 1610 & 1626 \\
\hline 25 & 8 & 10 & 1248 & 2333 & 2448 & 2448 \\
\hline 25 & 8 & 11 & 1662 & 3422 & 3575 & 3575 \\
\hline 25 & 8 & 12 & 2576 & 4087 & 4169 & 4316 \\
\hline 26 & 8 & 9 & 883 & 2108 & 2160 & 2282 \\
\hline 26 & 8 & 10 & 1519 & 3496 & 3719 & 3719 \\
\hline 26 & 8 & 11 & 1988 & 5225 & 5315 & 5416 \\
\hline 26 & 8 & 12 & 3070 & 6741 & 6834 & 7634 \\
\hline 26 & 8 & 13 & 3588 & 7080 & 7164 & 8030 \\
\hline 27 & 8 & 10 & 1597 & 4986 & 5260 & 5260 \\
\hline 27 & 8 & 11 & 2295 & 7833 & 7837 & 8381 \\
\hline 27 & 8 & 13 & 4094 & 11981 & 11991 & 12883 \\
\hline 28 & 8 & 10 & 1820 & 7016 & 7368 & 7368 \\
\hline 28 & 8 & 12 & 4916 & 17011 & 17299 & 17299 \\
\hline 28 & 8 & 13 & 4805 & 21152 & 21739 & 21739 \\
\hline 28 & 8 & 14 & 6090 & 22710 & 23268 & 23268 \\
\hline 22 & 10 & 10 & 46 & 72 & 73 & 82 \\
\hline 22 & 10 & 11 & 46 & 80 & 81 & 88 \\
\hline 24 & 10 & 9 & 56 & 118 & 119 & 119 \\
\hline 25 & 10 & 11 & 125 & 380 & 388 & 388 \\
\hline 25 & 10 & 12 & 132 & 434 & 464 & 465 \\
\hline 26 & 10 & 10 & 130 & 406 & 410 & 412 \\
\hline 26 & 10 & 11 & 168 & 566 & 581 & 621 \\
\hline 26 & 10 & 12 & 195 & 702 & 728 & 842 \\
\hline 26 & 10 & 13 & 210 & 754 & 869 & 897 \\
\hline 27 & 10 & 10 & 162 & 571 & 577 & 579 \\
\hline 27 & 10 & 11 & 222 & 882 & 900 & 1011 \\
\hline 27 & 10 & 12 & 351 & 1201 & 1289 & 1306 \\
\hline 27 & 10 & 13 & 405 & 1419 & 1460 & 1479 \\
\hline 28 & 10 & 11 & 286 & 1356 & 1434 & 1453 \\
\hline 28 & 10 & 12 & 365 & 1977 & 1981 & 1981 \\
\hline 25 & 12 & 10 & 28 & 37 & 38 & 40 \\
\hline 26 & 12 & 11 & 39 & 66 & 69 & 85 \\
\hline 26 & 12 & 13 & 58 & 91 & 92 & 106 \\
\hline 27 & 12 & 10 & 39 & 64 & 65 & 83 \\
\hline 28 & 12 & 10 & 49 & 87 & 99 & 105 \\
\hline
\end{tabular}

TABLE II

NEW UPPER BOUNDS ON $A(n, d, w)$

\section{REFERENCES}

[1] E. Agrell, A. Vardy, K. Zeger, "Upper bounds for constantweight codes," IEEE Trans. Inform. Theory, vol. IT-46, pp. 2373-2395, November 2000.

[2] E. Agrell, A. Vardy, K. Zeger, "A table of upper bounds for binary codes," IEEE Trans. Inform. Theory, vol. IT-47, pp. 3004-3006, November 2001.

[3] M.R. Best, A.E. Brouwer, F.J. MacWilliams, A.M. Odlyzko, N.J.A. Sloane, "Bounds for binary codes of length less than 25," IEEE Trans. Inform. Theory, vol. IT-24, pp. 81-93, January 1978.

[4] P. Delsarte, "An algebraic approach to the association schemes of coding theory," Philips Res. Repts. Suppl., no. 10, 1973.

[5] K. Elssel, K.-H. Zimmermann, "Two new nonlinear binary codes," IEEE Trans. Inform. Theory, vol IT-51, pp. 1189-1190, March 2005.

[6] J.T. Go, "The Terwilliger algebra of the hypercube," European J. Combin., vol. 23, pp. 399-429, May 2002.

[7] L. Lovász, "On the Shannon capacity of a graph," IEEE Trans. Inform. Theory, vol. IT-25, pp. 1-7, January 1979.

[8] L. Lovász, A. Schrijver, "Cones of matrices and set-functions and 0-1 optimization," SIAM J. Optim., vol. 1, pp. 166-190, May 1991.

[9] F.J. MacWilliams, N.J.A. Sloane, The Theory of ErrorCorrecting Codes, Amsterdam, The Netherlands: NorthHolland, 1977.

[10] R.J. McEliece, E.R. Rodemich, H.C. Rumsey, Jr, "The Lovász bound and some generalizations," J. Combin. Inform. System Sci., vol. 3 (no. 3), pp. 134-152, 1978.

[11] B. Mounits, T. Etzion, S. Litsyn, "Improved upper bounds on sizes of codes," IEEE Trans. Inform. Theory, vol. IT-48, pp. 880-886, April 2002.

[12] P.R.J. Östergård, "Two new four-error-correcting binary codes," preprint, 2003. Available: http://www.hut.fi/ pat/papers/four.ps

[13] A. Schrijver, "A comparison of the Delsarte and Lovász bounds," IEEE Trans. Inform. Theory, vol. IT-25, pp. 425-429, July 1979.

[14] P. Terwilliger, "The subconstituent algebra of an association scheme (Part I)," J. Algebraic Combin., vol. 1, pp. 363-388, December 1992.

[15] M.J. Todd, "Semidefinite optimization," Acta Numer., vol. 10, pp. 515-560, May 2001.

[16] R.H. Tütüncü, K.C. Toh, M.J. Todd, "Solving semidefinitequadratic-linear programs using SDPT3," Math. Program., Series $B$, vol. 95, pp. 189-217, February 2003.

[17] S.J. Wright, "Nonlinear and semidefinite programming," in: Trends in Optimization, Proc. Sympos. Appl. Math., 61, Providence, RI: Amer. Math. Soc., 2004, pp. 115-137. 